\title{
LA RÉSISTIVITÉ ÉLECTRIQUE COMME OUTIL POUR LA RECONNAISSANCE D'UNE FRICHE INDUSTRIELLE
}

\author{
Roger Guérin* et**, Yves Benderitter*, Jacques David* et Alain Tabbagh* \\ *UMR 7619 Sisyphe, Université Pierre et Marie Curie (Paris 6)
}

Le site de la friche industrielle de Mortagne-du-Nord (France) a abrité des activités de métallurgie de zinc et de plomb et de fabrication d'acide sulfurique. Des études hydrologiques et géochimiques y ont été menées dans des fosses et des forages après que le site ait été rasé et recouvert par des remblais. Les données géophysiques (cartographie électromagnétique slingram, panneau électrique 2D et 3D, et diagraphie électrostatique) permettent de localiser les sols et les eaux minéralisés, de positionner les interfaces entre couches, d'identifier les lieux de sortie de l'eau vers les drains fluviaux et de caractériser la zone non-saturée, avec une bonne résolution spatiale.

The industrial waste land in Mortagne-du-Nord (France) has been occupied by a zinc and a lead smelter unit linked to sulphuric acid production. Then this factory has been razed and covered by coarse waste deposits below sandy fillings. Hydrological and geochemical studies have been systematically carried out by drillings and pits following a mesh of $50 \mathrm{~m} \times 50 \mathrm{~m}$. This waste land constitutes an area where the metallic contamination is important. The physical property of subsoil materials, sensitive to this contamination, is the electrical resistivity; it depends on water mineralization and quantity presented in all formations. Geophysical data allow to locate precisely mineralised soil and water with higher spatial resolution. A complete geophysical study, using methods based on electrical resistivity (slingram electromagnetic mapping, 2D and 3D electrical imaging, and electrostatic logging) has been carried out. The slingram mapping allows to determine conductive anomalies coincident with chemical anomalies obtained into the piezometers, and to locate the water seepage toward the river drain. The electrical imaging allows to indicate precisely the location of clayey alluvial deposits, and to describe the superficial seepage areas. The electrostatic logging gives an accurate description of the not saturated area in boreholes, near anomalies located with the two other methods. Those geophysical methodologies allow to obtain a 3D view of the site.

\section{INTRODUCTION}

De nombreuses friches industrielles et sites pollués présentent des eaux très acides contenant des ions métalliques toxiques de forte mobilité. Pour prévoir la remédiation de tels sites, il est nécessaire de localiser et de décrire avec des méthodes non-destructives les zones où se trouvent les concentrations (trop) élevées de pollution.

L'objectif de cette étude, financée par l'Ademe (contrat $\left.\mathrm{n}^{\circ} 0074054\right)$, est de définir une méthodologie de mesures géophysiques qui puisse être utilisée sur de tels sites afin : i) de cartographier la distribution des matériaux pollués, ii) de suivre le niveau piézomètrique et ses variations saisonnières, iii) d'évaluer la minéralisation de l'eau et ses variations spatiales, et iv) d'identifier les fuites d'eau en direction de drains quand elles existent.

\section{PRÉSENTATION DU SITE}

Le site de Mortagne-du-Nord (59, France), couvre une surface d'environ 25 ha. Entre 190I et 1968, une usine de métallurgie du zinc et du plomb, ainsi que de fabrication d'acide sulfurique a occupé le site. Ce site est situé entre deux drains, le canal de la Scarpe au nord-est et le Décours au sud-ouest (figure I).

Les usines avaient été construites sur une ancienne zone marécageuse. Elles ont été rasées et le site est maintenant recouvert par des remblais sableux et des remblais industriels (scories, creusets...) sur une épaisseur d'environ $2 \mathrm{~m}$. Le niveau piézomètrique est situé à l'intérieur des remblais, au-dessus de la couche argileuse qui correspond aux dépôts de l'ancien marais et qui constitue une limite a priori imperméable. Ces alluvions argileuses reposent sur un substratum constitué par les sables d'Ostricourt. L'aquifère s'écoule dans les deux rivières directement quand le niveau piézomètrique est haut, et par infiltration quand le niveau est bas (figure 2).

Des mesures géochimiques (figure 3), par prélèvements et forages, ont été effectuées dans une série de piézomètres pour étudier l'impact environnemental de ces déchets industriels [I]. Néanmoins, pour pouvoir 


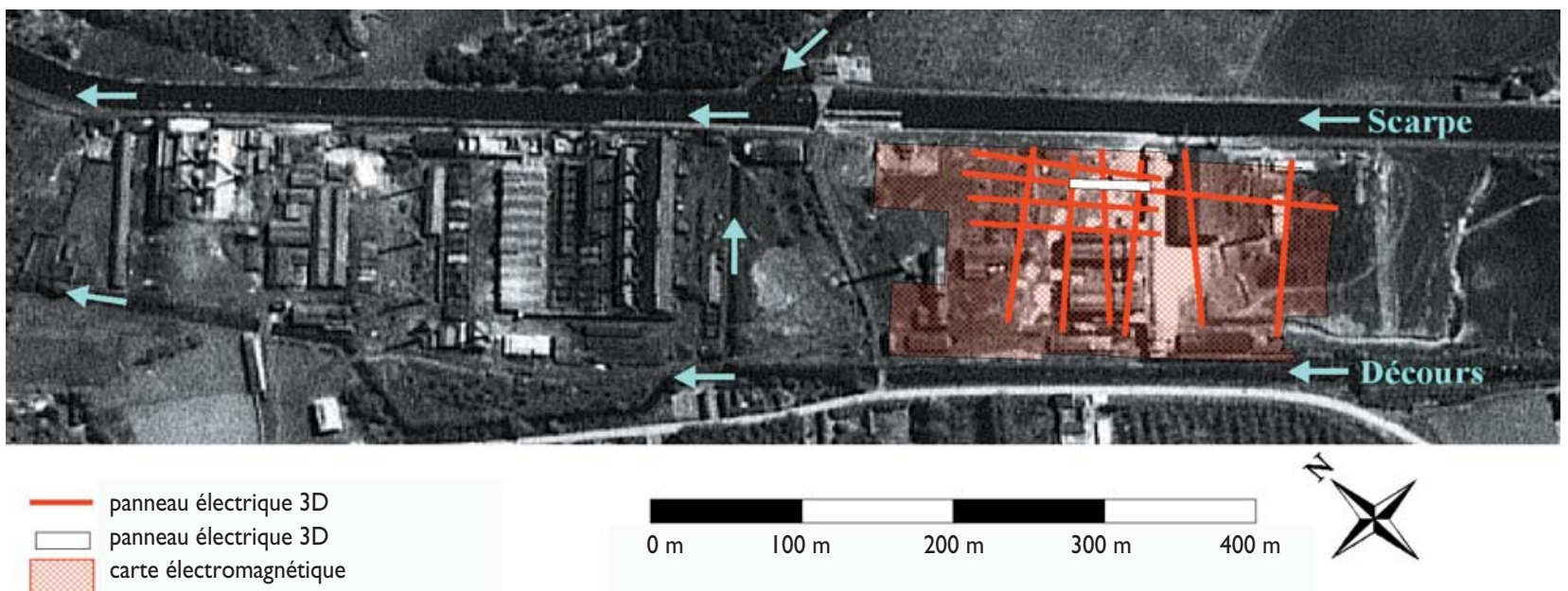

Figure I : Photographie aérienne de la friche industrielle de Mortagne-du-Nord avec position des prospections géophysiques

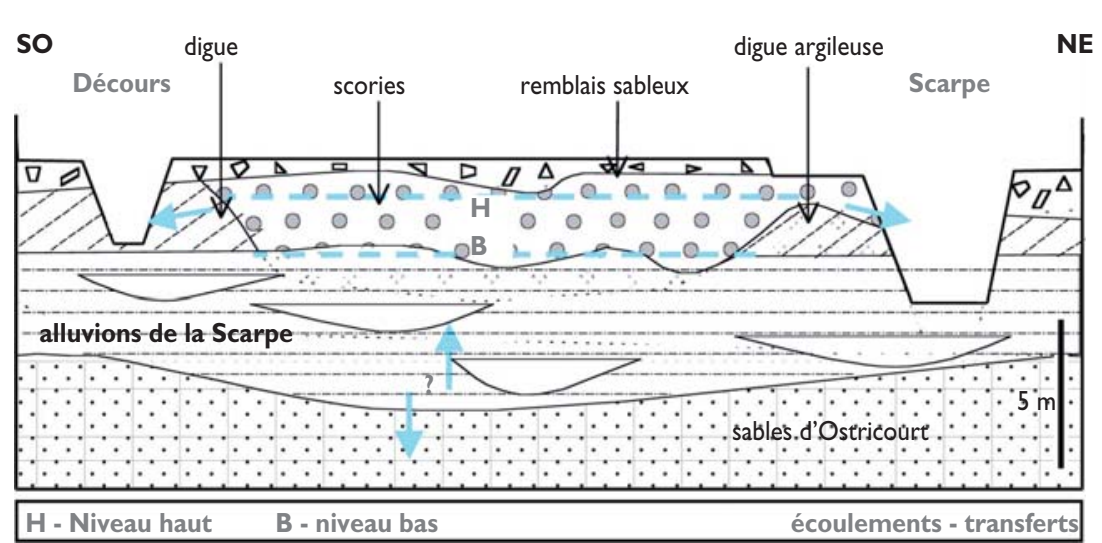

Figure 2 : Schéma de principe de l'hydrologie de la friche industrielle

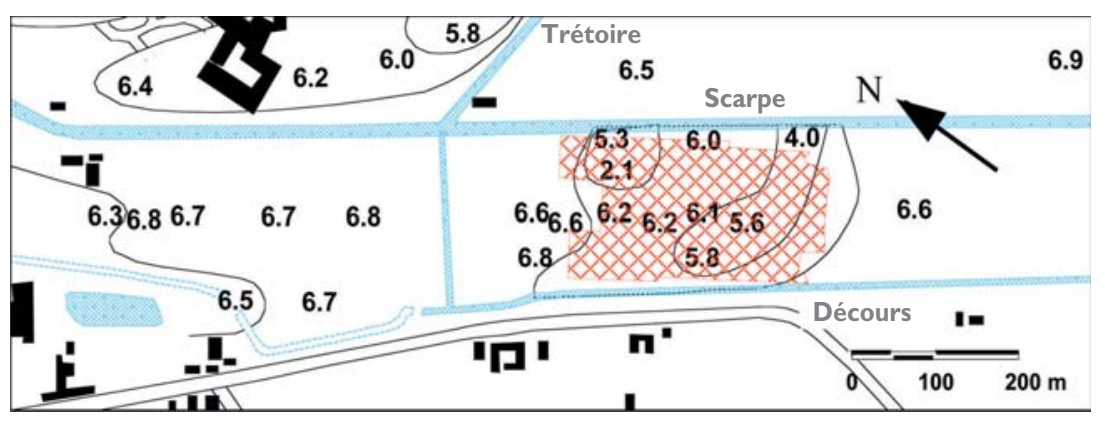

Figure 3 : pH mesurés sur les eaux de la nappe superficielle avec position des prospections géophysiques

prendre des mesures de protection sur le site, il est nécessaire de disposer d'une résolution spatiale plus grande qui permette de délimiter précisément les contours des zones polluées et d'identifier les zones de circulations préférentielles.

\section{MÉTHODOLOGIE}

L'objectif des prospections géophysiques sur cette friche industrielle ${ }^{22}$ est de décrire la nature et la géo- métrie des formations et les caractéristiques qui gouvernent les mouvements de fluide dans le sol : propriétés hydrauliques du sous-sol, teneur en eau et minéralisation. La résistivité électrique est la propriété physique la plus adaptée pour ce type d'étude en raison de sa dépendance avec les contenus en eau, en ions et en argile. Les méthodes géophysiques mesurant une résistivité électrique apparente (ou son inverse une conductivité) correspondant à la résistivité d'un certain volume de sol, sont donc à privilégier. La cartographie électromagnétique slingram [3], le panneau électrique $2 D$ et $3 D^{[4]}$ et la diagraphie électrostatique [5] sont donc bien adaptés dans ce contexte.

\section{RÉSULTATS}

La carte de conductivité électrique apparente slingram, obtenue avec l'appareil EM3I (Geonics), couvre une surface d'environ $300 \mathrm{~m}$ par $150 \mathrm{~m}$ suivant un maillage carré $5 \mathrm{~m}$ par $5 \mathrm{~m}$ (figure 4).

Elle donne sur les six premiers mètres de profondeur des informations sur : i) les variations en profondeur du toit de l'aquifère minéralisé, ii) les alluvions argileuses (associées aux anomalies conductrices), et iii) les sorties d'eau vers les drains fluviaux. Deux grandes zones apparaissent sur cette carte. La première relativement conductrice (en bleu/vert) correspond à une zone baignée par de l'eau fortement minéralisée reconnue par les études géochimiques (conductivité de l'ordre de $3000 \mu \mathrm{S} \mathrm{cm}-\mathrm{I}$ ), et aux alluvions argileuses proches de la surface. La secon- 
de relativement résistante (en rouge/marron) correspond soit à une couche non ou moins minéralisée, soit à des alluvions plus profondes. La répartition spatiale des piézomètres bien adaptée au suivi du niveau et de la qualité de l'eau de la nappe, est insuffisante pour caractériser la structure du sous-sol (il n'y a pas ainsi de piézomètres centrés sur les anomalies détectées par la géophysique).

Une série de panneaux électriques $2 \mathrm{D}$ a été réalisée, avec le système multi-électrodes d'Iris Instruments, suivant deux directions plus ou moins orthogonales afin de créer une grille irrégulière de maille approximative 25-50 m (figure 5). L'interprétation des différentes sections permet d'identifier trois couches principales ayant des gammes de résistivités bien différenciées qui correspondent à différents faciès géologiques. Ces trois faciès sont les suivants : i) un recouvrement résistant (en rouge/marron) superficiel qui correspond aux vestiges industriels (anciennes fondations, dalles de ciments des anciennes usines) et remblais minéralisés dont l'épaisseur varie entre 0,5 et $4 \mathrm{~m}$, ii) une couche conductrice (en bleu/vert) qui correspond aux alluvions argileuses à des profondeurs intermédiaires entre 4 et $12 \mathrm{~m}$ de profondeur, et iii) un substratum de résistivité moyenne (en jaune/orange) qui correspond aux sables d'Ostricourt. Les valeurs de résistivité interprétée, plus faible qu'attendues (10 au lieu de $20 \Omega . m$ pour les alluvions argileuses, et 50 au lieu de 200 S.m pour les sables), doivent être dues à la présence d'une eau minéralisée qui baigne toutes les formations. Par ailleurs, nous pouvons observer d'importantes variations latérales des épaisseurs/profondeurs des trois couches, ainsi que des anomalies superficielles (conductrices pour celles qui correspondent à des zones d'infiltration préférentielle, et résistantes pour celles qui correspondent aux anciennes structures construites).

La prospection par panneau électrique 3D a été effecrente wenner- $\alpha 4 \mathrm{~m}$ ) I m)

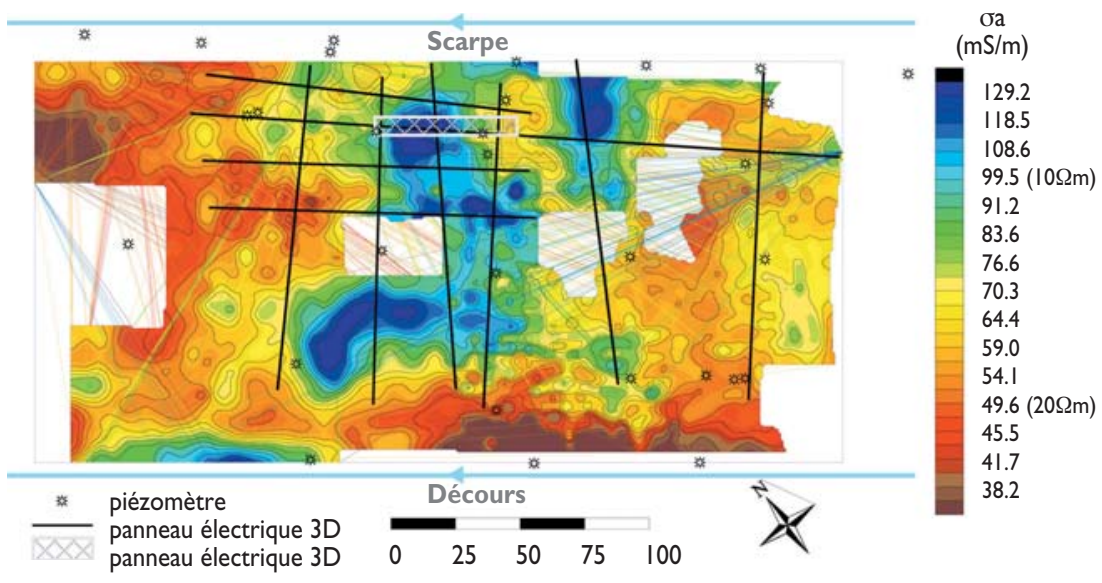

Figure 4 : Carte électromagnétique slingram de conductivité électrique appa-

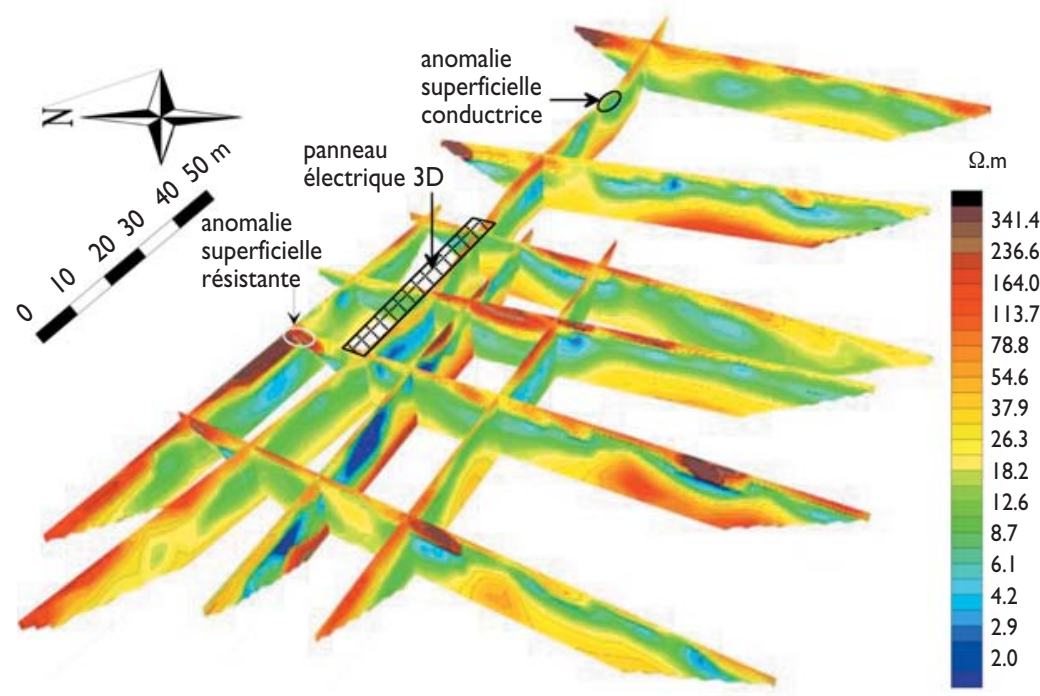

Figure 5 : Coupes verticales de résistivité électrique interprétée (dispositif

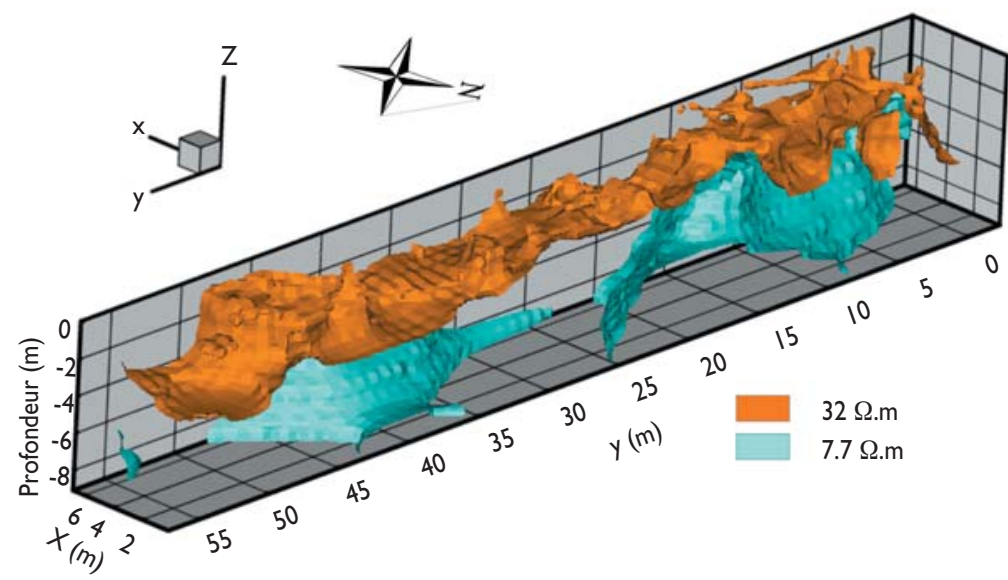

Figure 6 : Vue 3D de résistivité électrique interprétée (dispositif pôle-pôle

tuée avec un échantillonnage spatial très fin (distance inter-électrode de $\mathrm{I} \mathrm{m}$ ) et a permis de décrire les zones 
d'infiltration aux alentours des anomalies conductrices détectées par les cartographies (figure 6). Les deux surfaces iso-résistivités représentent l'interface entre les trois couches décrites précédemment.

Des diagraphies effectuées à l'intérieur de piézomètres permettent de caler et calibrer les données de géophysique avec la géologie. Ce calage indispensable, tout comme des relevés sur des anomalies localisées par la géophysique de surface, permet de vérifier la validité des interprétations géophysiques et de préciser l'origine des anomalies. Une sonde électrostatique, moins sensible aux perturbations métalliques qu'une sonde électromagnétique, et utilisable en milieu non-saturé comme en saturé, à la différence des sondes électriques classiques, est en cours de test.

\section{CONCLUSIONS}

Les résultats des données obtenues sur la friche industrielle de Mortagne-du-Nord montre l'intérêt des méthodes géophysiques pour identifier la structure spatiale d'un tel milieu hétérogène et complexe. Les prospections à différentes profondeurs et sur une large surface ont permis de localiser les zones d'eau à forte minéralisation, et d'identifier les fuites d'eau en direction des deux drains fluviaux. Les informations indispensables données par les forages sont complétées par celles fournies par la géophysique qui permettent de les interpoler tout en offrant la possibilité d'atteindre une échelle d'analyse métrique. Les méthodes géophysiques ayant pour cible la résistivité électrique peuvent être utilisées seules ou combinées, pas seulement sur des sites industriels et miniers, mais aussi sur des sites de déchets ménagers.

Roger Guérin *, **, Yves Benderitter *, Jacques David * et Alain Tabbagh *

* UMR 7619 Sisyphe, Département de géophysique appliquée, Université Pierre et Marie Curie (Paris 6), case 105, 4 place Jussieu, 75252 Paris Cedex 05

** Tél. : 0 I 442745 9I, fax : 01442745 88, mail : guerin@ccr.jussieu.fr

\section{Remerciements}

Ce travail a bénéficié d'un soutien financier de l'Ademe (contrat n0074054). Les auteurs tiennent à remercier également $\mathrm{P}$. Begassat (Ademe) pour son soutien et ses remarques scientifiques, $M$. Thiry (Ecole des mines de Paris, Centre informatique géologique, UMR 7619 Sisyphe) pour sa collaboration et les mesures géochimiques, et N. Bouzid, K. Chalikakis, A. Chotard, C. Panissod pour leur contribution à l'acquisition et au traitement des données.

\section{Références}

[I] Thiry M., Huet-Taillanter S. et Schmitt J.M. (2002) La friche industrielle de Mortagne-du-Nord (59) -

- Prospection du site, composition des scories, hydrochimie, hydrologie et estimation des flux. Bulletin de la Société Géologique de France, 173 (4) 369-381.

[2] Guérin R., Panissod C., Thiry M. Benderitter Y., Tabbagh A. et HuetTaillanter S. (2002) La friche industrielle de Mortagne-du-Nord (59) - III Approche méthodologique d'étude géophysique non-destructive des sites pollués par des eaux fortement minéralisées. Bulletin de la Société Géologique de France, I73 (5), 47I-477.
[3] McNeill J.D. (1980) Electromagnetic terrain conductivity measurement at low induction numbers. Technical Note TN-6, Geonics Limited, I5 p.

[4] Loke M.H. and Barker R.D. (1996) Rapid least-square inversion of apparent resistivity pseudosections by a quasi-Newton method. Geophysical Prospecting, 44, |3|-| 52.

[5] Tabbagh A. and Panissod C. (200I) ID complete calculation for electrostatic soundings interpretation. Geophysical Prospecting, 48, 51 I-520. 